\title{
0310 THE MAGNITUDE OF THE MOTOR VEHICLE CRASHES IN THE UNITED ARAB EMIRATES DURING THE PERIOD 1978-2007
}

Y Al Hosani*, M Stevenson, M El Sadig Correspondence: UAEArmed Forces Medical Services Corps, Directorate of Public Health and Preventive Medicine, PO Box 6723, Abu Dhabi, United Arab Emirates

\subsection{6/ip.2010.029215.310}

The WHO indicates that annually over one million people die and between 20 and 50 million people are injured in motor vehicle collisions (MVCs) worldwide (Peden et al, 2004).

In the United Arab Emirates (UAE) motor vehicle crashes are the second leading cause of death accounting for $12.3 \%$ of all deaths in 2007 (Ministry of Health (MOH), 2007).

A retrospective analysis was conducted based on secondary data obtained from the UAE Ministry of Interior (MOI), Abu Dhabi Police and $\mathrm{MOH}$ official reports during the period 1977-2007.

During the period 1977-2005, more than 400000 motor vehicle crashes occurred in the UAE, resulting in 15236 deaths and 233111 injured persons (MOI, 1977-2005).

Drivers accounted for $49 \%$ and $25 \%$, passengers for $44 \%$ and $44 \%$ while pedestrians accounted for $23 \%$ and $12 \%$ of MVC fatalities and injuries, respectively. More than $41 \%$ of deaths occurred among the age group 18-45 years.

Although there was a threefold increase in the number of MVC deaths and injuries during the period 1978-2007, the death rate $/ 100000$ population declined by $40 \%$ from 37 in 1978 to 22 in 2007 and death rate per 100000 registered vehicle declined by $86 \%$ from 382 to 55 during the same period.

The road traffic safety situation in the UAE is unacceptable when compared to high income countries (Bener and Alwash, 2002). Thus, serious steps with a bundle of comprehensive, multifaceted interventions that have been shown to be effective elsewhere should be adapted and implemented. 